\title{
Neuropilin-1 facilitates SARS-CoV-2 cell entry and infectivity
}

\author{
Ludovico Cantuti-Castelvetri1,2*, Ravi Ojha ${ }^{3 *}$, Liliana D. Pedro ${ }^{1,2 *}$, Minou Djannatian $^{1,2 *}$, Jonas Franz ${ }^{4 *}$, Suvi \\ Kuivanen $^{5 *}$, Franziska van der Meer ${ }^{4}$, Katri Kallio ${ }^{3}$, Tuğberk Kaya ${ }^{1,2,6}$, Maria Anastasina ${ }^{3,7}$, Teemu Smura ${ }^{5}$, Lev \\ Levanov $^{5}$, Leonora Szirovicza ${ }^{5}$, Allan Tobi ${ }^{8}$, Hannimari Kallio-Kokko ${ }^{9}$, Pamela Österlund ${ }^{10}$, Merja Joensuu ${ }^{11}$, \\ Frédéric A. Meunier ${ }^{11}$, Sarah J. Butcher ${ }^{3,7}$, Martin Sebastian Winkler ${ }^{12}$, Brit Mollenhauer ${ }^{13}$, Ari Helenius ${ }^{14}$, \\ Ozgun Gokce $^{6,19}$, Tambet Teesalu ${ }^{3,15,16}$, Jussi Hepojoki ${ }^{5,17}$, Olli Vapalahti ${ }^{5,9,18}$, Christine Stadelmann ${ }^{4}$, Giuseppe \\ Balistreri $^{3,19}+$, Mikael Simons ${ }^{1,2,20} \uparrow$
}

\begin{abstract}
${ }^{1}$ Institute of Neuronal Cell Biology, Technical University Munich, Munich, Germany. ${ }^{2}$ German Center for Neurodegenerative Diseases (DZNE), Munich, Germany. ${ }^{3}$ Faculty of Biological and Environmental Sciences, Molecular and Integrative Biosciences Research Program, University of Helsinki, Helsinki, Finland. ${ }^{4}$ Department of Neuropathology, University Medical Center Göttingen, Göttingen, Germany. ${ }^{5}$ University of Helsinki, Medicum, Department of Virology, Helsinki, Finland. ${ }^{6}$ Institute for Stroke and Dementia Research (ISD), University Hospital, LMU Munich, Munich, Germany. ${ }^{7}$ Helsinki Institute of Life Sciences-Institute of Biotechnology, University of Helsinki, Finland. ${ }^{8}$ Laboratory of Cancer Biology, Institute of Biomedicine and Translational Medicine, University of Tartu, Tartu, Estonia. ${ }^{9}$ University of Helsinki and Helsinki University Hospital, Department of Virology, Helsinki, Finland. ${ }^{10}$ Department of Health Security, Finnish Institute for Health and Welfare (THL), Helsinki, Finland. ${ }^{11} \mathrm{Clem}$ Jones Centre for Ageing Dementia Research, Queensland Brain Institute, The University of Queensland, Brisbane, Queensland, Australia. ${ }^{2}$ Department of Anesthesiology and Intensive Care Medicine, University Medical Center Göttingen, Göttingen, Germany. ${ }^{13}$ Department of Neurology, University Medical Center Göttingen, Göttingen, Germany \& Paracelsus-Elena-Klinik Kassel, Kassel, Germany. ${ }^{14}$ Institute of Biochemistry, ETH Zürich, Zürich, Switzerland. ${ }^{15}$ Cancer Research Center, Sanford Burnham Prebys Medical Discovery Institute, La Jolla, California, USA. ${ }^{16}$ Center for Nanomedicine and Department of Cell, Molecular and Developmental Biology, University of California, Santa Barbara, Santa Barbara, California, USA. ${ }^{17}$ Institute of Veterinary Pathology, Vetsuisse Faculty, University of Zürich, Zürich, Switzerland. ${ }^{18}$ Dept of Veterinary Biosciences, University of Helsinki, Helsinki, Finland. ${ }^{19}$ The Queensland Brain Institute, University of Queensland, Brisbane, Australia. ${ }^{20}$ Munich Cluster of Systems Neurology (SyNergy), Munich, Germany.
\end{abstract}

*These authors contributed equally to this work.

†Corresponding author. Email: giuseppe.balistreri@helsinki.fi (G.B.); mikael.simons@dzne.de (M.S.)

The causative agent of coronavirus induced disease 2019 (COVID-19) is the severe acute respiratory syndrome coronavirus 2 (SARS-CoV-2). For many viruses, tissue tropism is determined by the availability of virus receptors and entry cofactors on the surface of host cells. Here, we found that neuropilin-1 (NRP1), known to bind furin-cleaved substrates, significantly potentiates SARS-CoV-2 infectivity, an effect blocked by a monoclonal blocking antibody against NRP1. A SARS-CoV-2 mutant with an altered furin cleavage site did not depend on NRP1 for infectivity. Pathological analysis of human COVID-19 autopsies revealed SARSCoV-2 infected cells including olfactory neuronal cells facing the nasal cavity positive for NRP1. Our data provide insight into SARS-CoV-2 cell infectivity and define a potential target for antiviral intervention.

An outbreak of SARS-CoV-2 infections has caused a pandemic associated with a severe acute pulmonary disease named COVID-19 (coronavirus induced disease 2019) (1). A related coronavirus, SARS-CoV, led to a much smaller outbreak in 2003, possibly due to infection occurring predominantly in the lower respiratory system, whereas SARS-CoV-2 spreads rapidly through active pharyngeal viral shedding (2). Despite these differences, uptake of both viruses is mediated by the identical cellular receptor, angiotensin-converting enzyme 2 (ACE2) (3-5). One attractive hypothesis to explain the enhanced spreading of SARS-CoV-2 is the presence of a polybasic furin-type cleavage site, $\mathrm{RRAR}^{\wedge} \mathrm{S}$, at the $\mathrm{S} 1 / \mathrm{S} 2$ junction in the SARS-CoV-2 spike (S) protein that is absent in SARS-CoV (6). Similar sequences are found in the S proteins of many other pathogenic human viruses, including Ebola, HIV-1 and highly virulent strains of avian influenza $(6,7)$. The presence of the polybasic cleavage site in SARS-CoV-2 results in enhanced pathogenicity by priming the fusion activity (8) and could potentially create additional cell surface receptor binding sites. Proteolytic cleavage of $\mathrm{RRAR}^{\wedge} \mathrm{S}$ by furin exposes a conserved carboxyterminal (C-terminal) motif $\mathrm{RXXR}_{\mathrm{OH}}$ (where $\mathrm{R}$ is arginine and $\mathrm{X}$ is any amino acid; $\mathrm{R}$ can be substituted by lysine, $\mathrm{K}$ ) in the $\mathrm{S}$ protein. Such C-terminal sequences that conform to the "C-end rule" (CendR) are known to bind to and activate neuropilin (NRP1 and NRP2) receptors at the cell surface (9). Recent cryo-electron microscopy structures of the SARS-CoV-2 S protein demonstrated that the $\mathrm{S} 1 / \mathrm{S} 2$ junction is part of a solventexposed loop and therefore accessible for receptor interactions $(10,11)$.

To determine whether SARS-CoV-2 can use NRP1 for virus entry and infectivity, we generated lentiviral particles pseudotyped with the SARS-CoV-2 S protein. Pseudoviruses are well suited for virus entry assays, as they allow viral entry 
to be distinguished from other virus life cycle steps. HEK293T cells, which have almost no detectable ACE2 and NRP1 transcripts (fig. S1), were transfected with plasmids encoding the two established host factors (4), human ACE2 and the transmembrane protease serine 2 (TMPRSS2), or NRP1. When expressed alone, ACE2 rendered cells susceptible to infection (Fig. 1A). While NRP1 only hardly promoted infection in HEK-293T cells, its co-expression with ACE2 and TMPRSS2 markedly enhanced infection (Fig. 1, A and B). NRP1 expression increased infection in Caco-2 cells, which endogenously express ACE2 (12) (Fig. 1C and fig. S1D), showing that NRP1 can potentiate infection in the presence of other host factors. To test the specificity of NRP1-dependent virus entry, we developed monoclonal antibodies (mAbs) that were designed to functionally block the extracellular b1b2 domain of NRP1, known to mediate the binding to CendR peptides (13). The mAb3 was observed to bind to the recombinant b1b2 domain of wild-type NRP1, but not to the triple mutant b1b2 domain (S346A, E348A and T349A in the CendR binding pocket) (fig. S2A). The potency of the mAbs in preventing cellular binding and internalization of NRP ligands was tested using $80 \mathrm{~nm}$ silver nanoparticles (AgNP) coated with the prototypic NRP1-binding CendR peptide RPARPAR $\mathrm{OH}_{\mathrm{OH}}$ (9) (fig. S2B). mAb3 efficiently blocked AgNP-CendR binding (fig. S2C) and internalization (fig. S2, D and E), while another monoclonal antibody, mAb2, had no effect and was used as a control in further experiments. Treatment of HEK-293T with mAb3 significantly reduced infection by SARS-CoV-2 pseudoviruses in cells expressing ACE2/TMPRSS2/NRP1 (Fig. 1D), but not in cells expressing ACE2/TMPRSS2 only (fig. S2F). When SARS-CoV-2 pseudovirus was pre-incubated with recombinant, soluble extracellular b1b2 domain of NRP1, the wild-type significantly reduced infection compared to the triple mutant (Fig. 1E and fig. S2G).

Next, we explored the role of NRP1 using SARS-CoV-2 isolated from COVID-19 patients from the Helsinki University Hospital. We used wild-type SARS-CoV-2 and a cleavage-impaired SARS-CoV-2 mutant that was isolated from Vero-E6 which rapidly accumulate mutations at the furin cleavage site of the S protein over passaging (Fig. 2, A and B) (14). First, we confirmed that furin cleaved the wild-type, but not the mutant, SARS-CoV-2 S protein by analyzing S protein processing in $\mathrm{CHO}$ cells with functional (parental) or deficient (FD11) furin enzyme (fig. S3) (15). Next, we validated that exogenous ACE2 expression rendered HEK-293T cells susceptible to infection with SARS-CoV-2 (Fig. 2, C and D). NRP1 expression alone caused lower levels of infection, which were only detectable with increasing virus titer (Fig. 2, C and D). We then compared the ability of wild-type and mutant SARS-CoV-2 virus to infect HEK-293T stably expressing either ACE2, ACE2/TMPRSS2 or ACE2/TMPRSS2/NRP1. Infection of these cell lines by the wild-type, but not the mutant, virus increased in the presence of NRP1, providing further evidence that NRP1 requires a furin-cleaved substrate for its effects (Fig. 2, E and F). Next, we studied the effect of the NRP1-blocking antibody, mAb3, on infection of Caco-2 cells by wild-type and mutant SARS-CoV-2 virus, and found that pre-incubation with NRP1-blocking antibody reduced wild-type virus infection by $\sim 40 \%$, while the control mAb2 had no effect (Fig. $2, \mathrm{G}$ and $\mathrm{H}$ ). NRP1-blocking antibody had no effect on the infection by the mutated virus (Fig. 2, G and $\mathrm{H}$ ).

Cleavage of SARS-CoV-2 S protein at the S1/S2 site generates the C-terminal end TQTNSPRRAR $\mathrm{OH}_{\mathrm{OH}}$. To determine whether this specific sequence can function as a substrate for NRP1, we used AgNPs coated with TQTNSPRRAR ${ }_{\mathrm{OH}}$ peptide or different control peptides including one with a terminal amide group, which reduces NRP1 binding (TQTNSPRRAR $_{\mathrm{NH} 2}$ ) (9) (Fig. 3A). We found that AgNPTQTNSPRRAR $_{\mathrm{OH}}$, but not control AgNPs were efficiently taken up by HEK-293T cells expressing NRP1 (Fig. 3, B and C). Next, we determined whether AgNP-TQTNSPRRAR ${ }_{\text {Oн }}$ particles were also internalized into cells in vivo. We chose to study nanoparticle entry in the mouse olfactory epithelium, owing to the known expression of NRP1 in the olfactory system (16) including olfactory neuronal cells of the epithelium (fig. S4). AgNPs-TQTNSPRRAR $\mathrm{OH}_{\mathrm{H}}$ and control AgNPTQTNSPRRAR $_{\mathrm{NH} 2}$ were administered into the nose of anesthetized adult mice. 6 hours after administration, we observed a significantly larger uptake of AgNP-TQTNSPRRAR ${ }_{\mathrm{OH}}$ compared to AgNP-TQTNSPRRAR $\mathrm{N}_{\mathrm{NH} 2}$ into the olfactory epithelium (Fig. 3, D and E), and unexpectedly also into neurons and blood vessels of the cortex (Fig. 3, F and G). Similar results were obtained for AgNPs coated with the prototypic NRP1-binding CendR peptide RPARPAR ${ }_{\mathrm{OH}}$ (fig. S5).

Having obtained evidence for a role of NRP1 in cell entry of SARS-CoV-2, we examined whether NRP1 expression correlated with the detection of virus RNA in single cell transcriptomes. For these analyses, we used published scRNA-seq datasets of cultured experimentally infected human bronchial epithelial cell (HBECs) and cells isolated from bronchoalveolar lavage fluid (BALF) of severely affected COVID19 patients (17). Among the proposed entry and amplification factors, NRP1, FURIN and TMPRSS11A, were enriched in SARS-CoV-2 infected cells compared to non-infected cells (fig. S6). We also detected increased expression of these proteins following infection (fig. S6). In addition, RNA expression of NRP1 and its homolog NRP2 was elevated in SARS-CoV-2positive cells compared to adjacent cells in the BALF of severely affected COVID-19 patients (fig. S7).

Because the availability of virus receptors and entry cofactors on the surface of host cells determines infectivity, we compared the expression patterns of $A C E 2$ and NRP1 in published scRNA-seq datasets of human lung tissue (18) and human olfactory epithelium (19). While ACE2 was detected at 
very low levels, both NRP1 and NRP2 were abundantly expressed in almost all pulmonary and olfactory cells with the highest expression in endothelial cells (figs. S8 and S9). We confirmed these results by examining NRP1 immunoreactivity in human autopsy tissue and detected NRP1 in the epithelial surface layer of the human respiratory and olfactory epithelium (fig. S10A). ACE2 was hardly detectable in these tissues (fig. S10B). Within the olfactory epithelium, NRP1 was also observed in late olfactory neuronal progenitors and/or newly differentiated olfactory neurons (fig. S10C).

In light of the widely reported disturbance of olfaction in a large fraction of COVID-19 patients (20) and the enrichment of NRPs in the olfactory epithelium, we analyzed a series of autopsies from six COVID-19 patients and eight non-infected control patients to determine whether SARS-CoV-2 could infect NRP1-positive cells (Fig. 4 and table S1). Using antibodies against the S protein, we detected infection of the olfactory epithelium in five out of six COVID-19 patients. The infected olfactory epithelial cells showed high expression of NRP1 (Fig. 4, A and B). Additional co-staining indicated infection of NRP1positive late olfactory neuronal progenitors and/or newly differentiated olfactory neurons (Fig. 4B and fig. S11).

There is limited knowledge about the virus-host interactions that determine cellular entry of SARS-CoV-2. Viruses display considerable redundancy and flexibility because they can exploit weak multivalent interactions to enhance affinity. While the focus to date has been almost entirely on the role of ACE2 in SARS-CoV-2 entry, the expression pattern of ACE2 does not match tissue tropism of SARS-CoV-2 (21). This raises the possibility that co-factors are required to facilitate virushost cell interactions in cells with low ACE2 expression. NRP1 could represent such an ACE2 potentiating factor; however, it is also possible that SARS-CoV-2 can enter cells independently of ACE2 when viral loads are high. The reason why a number of viruses (22-25) use NRPs as entry factors could be because of their high expression on epithelia facing the external environment, and their function in enabling cell, vascular, and tissue penetration $(9,13)$.

\section{REFERENCES AND NOTES}

1. N. Zhu, D. Zhang, W. Wang, X. Li, B. Yang, J. Song, X. Zhao, B. Huang, W. Shi, R. Lu, P. Niu, F. Zhan, X. Ma, D. Wang, W. Xu, G. Wu, G. F. Gao, W. Tan; China Novel Coronavirus Investigating and Research Team, A Novel Coronavirus from Patients with Pneumonia in China, 2019. N. Engl. J. Med. 382, 727-733 (2020). doi:10.1056/NEJMoa2001017 Medline

2. R. Wölfel, V. M. Corman, W. Guggemos, M. Seilmaier, S. Zange, M. A. Müller, D. Niemeyer, T. C. Jones, P. Vollmar, C. Rothe, M. Hoelscher, T. Bleicker, S. Brünink, J. Schneider, R. Ehmann, K. Zwirglmaier, C. Drosten, C. Wendtner, Virological assessment of hospitalized patients with COVID-2019. Nature 581, 465-469 (2020). doi:10.1038/s41586-020-2196-x Medline

3. P. Zhou, X.-L. Yang, X.-G. Wang, B. Hu, L. Zhang, W. Zhang, H.-R. Si, Y. Zhu, B. Li, C.L. Huang, H.-D. Chen, J. Chen, Y. Luo, H. Guo, R.-D. Jiang, M.-Q. Liu, Y. Chen, X.-R. Shen, X. Wang, X.-S. Zheng, K. Zhao, Q.-J. Chen, F. Deng, L.-L. Liu, B. Yan, F.-X. Zhan, Y.-Y. Wang, G.-F. Xiao, Z.-L. Shi, A pneumonia outbreak associated with a new coronavirus of probable bat origin. Nature 579, 270-273 (2020). doi:10.1038/s41586-020-2012-7 Medline
4. M. Hoffmann, H. Kleine-Weber, S. Schroeder, N. Krüger, T. Herrler, S. Erichsen, T. S. Schiergens, G. Herrler, N.-H. Wu, A. Nitsche, M. A. Müller, C. Drosten, S. Pöhlmann, SARS-CoV-2 Cell Entry Depends on ACE2 and TMPRSS2 and Is Blocked by a Clinically Proven Protease Inhibitor. Cell 181, 271-280.e8 (2020). doi:10.1016/i.cell.2020.02.052 Medline

5. N. J. Matheson, P. J. Lehner, How does SARS-CoV-2 cause COVID-19? Science 369, 510-511 (2020). doi:10.1126/science.abc6156 Medline

6. B. Coutard, C. Valle, X. de Lamballerie, B. Canard, N. G. Seidah, E. Decroly, The spike glycoprotein of the new coronavirus 2019-nCoV contains a furin-like cleavage site absent in CoV of the same clade. Antiviral Res. 176, 104742 (2020). doi:10.1016/j.antiviral.2020.104742 Medline

7. L. V. Tse, A. M. Hamilton, T. Friling, G. R. Whittaker, A novel activation mechanism of avian influenza virus H9N2 by furin. J. Virol. 88, 1673-1683 (2014). doi:10.1128/JVI.02648-13 Medline

8. M. Hoffmann, H. Kleine-Weber, S. Pöhlmann, A Multibasic Cleavage Site in the Spike Protein of SARS-CoV-2 Is Essential for Infection of Human Lung Cells. Mol. Cell 78, 779-784.e5 (2020). doi:10.1016/j.molcel.2020.04.022 Medline

9. T. Teesalu, K. N. Sugahara, V. R. Kotamraju, E. Ruoslahti, C-end rule peptides mediate neuropilin-1-dependent cell, vascular, and tissue penetration. Proc. Natl. Acad. Sci. U.S.A. 106, 16157-16162 (2009). doi:10.1073/pnas.0908201106 Medline

10. D. Wrapp, N. Wang, K. S. Corbett, J. A. Goldsmith, C.-L. Hsieh, O. Abiona, B. S. Graham, J. S. McLellan, Cryo-EM structure of the 2019-nCoV spike in the prefusion conformation. Science 367, 1260-1263 (2020). doi:10.1126/science. abb2507 Medline

11. A. C. Walls, Y.-J. Park, M. A. Tortorici, A. Wall, A. T. McGuire, D. Veesler, Structure, Function, and Antigenicity of the SARS-CoV-2 Spike Glycoprotein. Cell 181, 281292.e6 (2020). doi:10.1016/i.cell.2020.02.058 Medline

12. K. Liao, D. Sikkema, C. Wang, T. N. Lee, Development of an enzymatic assay for the detection of neutralizing antibodies against therapeutic angiotensin-converting enzyme 2 (ACE2). J. Immunol. Methods 389, 52-60 (2013). doi:10.1016/.j.jim.2012.12.010 Medline

13. A. Plein, A. Fantin, C. Ruhrberg, Neuropilin regulation of angiogenesis, arteriogenesis, and vascular permeability. Microcirculation 21, 315-323 (2014). doi:10.1111/micc.12124 Medline

14. A. D. Davidson, M. K. Williamson, S. Lewis, D. Shoemark, M. W. Carroll, K. J. Heesom, M. Zambon, J. Ellis, P. A. Lewis, J. A. Hiscox, D. A. Matthews, Characterisation of the transcriptome and proteome of SARS-CoV-2 reveals a cell passage induced in-frame deletion of the furin-like cleavage site from the spike glycoprotein. Genome Med. 12, 68 (2020). doi:10.1186/s13073-020-00763-0 Medline

15. N. G. Ravindra, M. M. Alfajaro, V. Gasque, J. Wei, R. B. Filler, N. C. Huston, H. Wan, K. Szigeti-Buck, B. Wang, R. R. Montgomery, S. C. Eisenbarth, A. Williams, A. M. Pyle, A. Iwasaki, T. L. Horvath, E. F. Foxman, D. van Dijk, C. B. Wilen, Single-cell longitudinal analysis of SARS-CoV-2 infection in human bronchial epithelial cells. bioRxiv 2020.05.06.081695 (2020). doi:10.1101/2020.05.06.081695

16. A. Kawakami, T. Kitsukawa, S. Takagi, H. Fujisawa, Developmentally regulated expression of a cell surface protein, neuropilin, in the mouse nervous system. $J$. Neurobiol. 29, 1-17 (1996). doi:10.1002/(SICl)1097-4695(199601)29:1<1:AlDNEU1>3.0.CO:2-F Medline

17. M. Liao, Y. Liu, J. Yuan, Y. Wen, G. Xu, J. Zhao, L. Cheng, J. Li, X. Wang, F. Wang, L. Liu, I. Amit, S. Zhang, Z. Zhang, Single-cell landscape of bronchoalveolar immune cells in patients with COVID-19. Nat. Med. 26, 842-844 (2020). doi:10.1038/s41591-020-0901-9 Medline

18. X. Han, Z. Zhou, L. Fei, H. Sun, R. Wang, Y. Chen, H. Chen, J. Wang, H. Tang, W. Ge, Y. Zhou, F. Ye, M. Jiang, J. Wu, Y. Xiao, X. Jia, T. Zhang, X. Ma, Q. Zhang, X. Bai, S. Lai, C. Yu, L. Zhu, R. Lin, Y. Gao, M. Wang, Y. Wu, J. Zhang, R. Zhan, S. Zhu, H. Hu, C. Wang, M. Chen, H. Huang, T. Liang, J. Chen, W. Wang, D. Zhang, G. Guo, Construction of a human cell landscape at single-cell level. Nature 581, 303-309 (2020). doi:10.1038/s41586-020-2157-4 Medline

19. M. A. Durante, S. Kurtenbach, Z. B. Sargi, J. W. Harbour, R. Choi, S. Kurtenbach, G. M. Goss, H. Matsunami, B. J. Goldstein, Single-cell analysis of olfactory neurogenesis and differentiation in adult humans. Nat. Neurosci. 23, 323-326 (2020). doi:10.1038/s41593-020-0587-9 Medline 
20. L. Mao, H. Jin, M. Wang, Y. Hu, S. Chen, Q. He, J. Chang, C. Hong, Y. Zhou, D. Wang, X. Miao, Y. Li, B. Hu, Neurologic Manifestations of Hospitalized Patients With Coronavirus Disease 2019 in Wuhan, China. JAMA Neurol. 77, 683-690 (2020). doi:10.1001/jamaneurol.2020.1127 Medline

21. F. Hikmet, L. Méar, A. Edvinsson, P. Micke, M. Uhlén, C. Lindskog, The protein expression profile of ACE2 in human tissues. Mol. Syst. Biol. 16, e9610 (2020). doi:10.15252/msb.20209610 Medline

22. D. Ghez, Y. Lepelletier, S. Lambert, J.-M. Fourneau, V. Blot, S. Janvier, B. Arnulf, P. M. van Endert, N. Heveker, C. Pique, 0 . Hermine, Neuropilin-1 is involved in human T-cell lymphotropic virus type 1 entry. J. Virol. 80, 6844-6854 (2006). doi:10.1128/JVL.02719-05 Medline

23. N. Martinez-Martin, J. Marcandalli, C. S. Huang, C. P. Arthur, M. Perotti, M. Foglierini, H. Ho, A. M. Dosey, S. Shriver, J. Payandeh, A. Leitner, A. Lanzavecchia, L. Perez, C. Ciferri, An Unbiased Screen for Human Cytomegalovirus Identifies Neuropilin-2 as a Central Viral Receptor. Cell 174, 1158-1171.e19 (2018). doi:10.1016/i.cell.2018.06.028 Medline

24. H. B. Wang, H. Zhang, J.-P. Zhang, Y. Li, B. Zhao, G.-K. Feng, Y. Du, D. Xiong, Q. Zhong, W.-L. Liu, H. Du, M.-Z. Li, W.-L. Huang, S. W. Tsao, L. Hutt-Fletcher, Y.-X. Zeng, E. Kieff, M.-S. Zeng, Neuropilin 1 is an entry factor that promotes EBV infection of nasopharyngeal epithelial cells. Nat. Commun. 6, 6240 (2015). doi:10.1038/ncomms 7240 Medline

25. M. Raaben, L. T. Jae, A. S. Herbert, A. I. Kuehne, S. H. Stubbs, Y. Y. Chou, V. A. Blomen, T. Kirchhausen, J. M. Dye, T. R. Brummelkamp, S. P. Whelan, NRP2 and CD63 Are Host Factors for Lujo Virus Cell Entry. Cell Host Microbe 22, 688-696.e5 (2017). doi:10.1016/i.chom.2017.10.002 Medline

26. H. Kleine-Weber, M. T. Elzayat, M. Hoffmann, S. Pöhlmann, Functional analysis of potential cleavage sites in the MERS-coronavirus spike protein. Sci. Rep. 8, 16597 (2018). doi:10.1038/s41598-018-34859-w Medline

27. B. G. Dorner, S. Steinbach, M. B. Hüser, R. A. Kroczek, A. Scheffold, Single-cell analysis of the murine chemokines MIP-1alpha, MIP-1beta, RANTES and ATAC/lymphotactin by flow cytometry. J. Immunol. Methods 274, 83-91 (2003). doi:10.1016/S0022-1759(02)00498-2 Medline

28. G. B. Braun, T. Friman, H.-B. Pang, A. Pallaoro, T. Hurtado de Mendoza, A.-M. A. Willmore, V. R. Kotamraju, A. P. Mann, Z.-G. She, K. N. Sugahara, N. O. Reich, T. Teesalu, E. Ruoslahti, Etchable plasmonic nanoparticle probes to image and quantify cellular internalization. Nat. Mater. 13, 904-911 (2014). doi:10.1038/nmat3982 Medline

29. I. Ullah, K. Chung, J. Beloor, S. K. Lee, P. Kumar, A Positioning Device for the Placement of Mice During Intranasal siRNA Delivery to the Central Nervous System. J. Vis. Exp. 2019, e59201 (2019). doi:10.3791/59201 Medline

30. C. T. Rueden, J. Schindelin, M. C. Hiner, B. E. DeZonia, A. E. Walter, E. T. Arena, K. W. Eliceiri, Image J2: Image J for the next generation of scientific image data. BMC Bioinformatics 18, 529 (2017). doi:10.1186/s12859-017-1934-z Medline

31. V. M. Corman, O. Landt, M. Kaiser, R. Molenkamp, A. Meijer, D. K. W. Chu, T. Bleicker, S. Brünink, J. Schneider, M. L. Schmidt, D. G. J. C. Mulders, B. L. Haagmans, B. van der Veer, S. van den Brink, L. Wijsman, G. Goderski, J.-L. Romette, J. Ellis, M. Zambon, M. Peiris, H. Goossens, C. Reusken, M. P. G. Koopmans, C. Drosten, Detection of 2019 novel coronavirus (2019-nCoV) by realtime RT-PCR. Euro Surveill. 25, 2000045 (2020). doi:10.2807/15607917.ES.2020.25.3.2000045 Medline

32. J. Quick, N. D. Grubaugh, S. T. Pullan, I. M. Claro, A. D. Smith, K. Gangavarapu, G. Oliveira, R. Robles-Sikisaka, T. F. Rogers, N. A. Beutler, D. R. Burton, L. L. LewisXimenez, J. G. de Jesus, M. Giovanetti, S. C. Hill, A. Black, T. Bedford, M. W. Carroll, M. Nunes, L. C. Alcantara Jr., E. C. Sabino, S. A. Baylis, N. R. Faria, M. Loose, J. T. Simpson, O. G. Pybus, K. G. Andersen, N. J. Loman, Multiplex PCR method for MinION and Illumina sequencing of Zika and other virus genomes directly from clinical samples. Nat. Protoc. 12, 1261-1276 (2017). doi:10.1038/nprot.2017.066 Medline

33. A. M. Bolger, M. Lohse, B. Usadel, Trimmomatic: A flexible trimmer for Illumina sequence data. Bioinformatics 30, 2114-2120 (2014). doi:10.1093/bioinformatics/btu170 Medline

34. H. Li, B. Handsaker, A. Wysoker, T. Fennell, J. Ruan, N. Homer, G. Marth, G. Abecasis, R. Durbin; 1000 Genome Project Data Processing Subgroup, The Sequence Alignment/Map format and SAMtools. Bioinformatics 25, 2078-2079 (2009). doi:10.1093/bioinformatics/btp352 Medline
35. A. Wilm, P. P. K. Aw, D. Bertrand, G. H. T. Yeo, S. H. Ong, C. H. Wong, C. C. Khor, R. Petric, M. L. Hibberd, N. Nagarajan, LoFreq: A sequence-quality aware, ultrasensitive variant caller for uncovering cell-population heterogeneity from highthroughput sequencing datasets. Nucleic Acids Res. 40, 11189-11201 (2012). doi:10.1093/nar/gks918 Medline

36. T. Stuart, A. Butler, P. Hoffman, C. Hafemeister, E. Papalexi, W. M. Mauck 3rd, Y. Hao, M. Stoeckius, P. Smibert, R. Satija, Comprehensive Integration of Single-Cell Data. Cell 177, 1888-1902.e21 (2019). doi:10.1016/i.cell.2019.05.031 Medline

\section{ACKNOWLEDGMENTS}

We thank René Müller, Katja Schulz and Uta Scheidt for expert technical assistance. Shona Osborne for proof-reading the manuscript, and DNA Dream Lab facility and Konstantin Kogan for design and cloning of plasmids. Funding: The work in Munich and Göttingen was supported by grants from the German Research Foundation (SPP2191, TRR128-2, TRR274-1, SyNergy Excellence Cluster, EXC2145, Projekt ID390857198, EXC 2067/1- 390729940, and STA 1389/5-1), the ERC (Consolidator Grant to M.S.), and the Dr. Miriam and Sheldon G. Adelson Medical Research. The work at the University of Helsinki was supported by the University of Helsinki and by donations of Finnish colleagues to whom we are very grateful. The academy of Finland supported G.B. (318434), O.V. (336490), S.J.B. (315950, and 336471), J.H. (1308613 and 1314119). O.V. was supported by Jane and Aatos Erkko Foundation and Helsinki University Hospital Funds (TYH2018322). S.J.B. was supported by the Swedish Research Foundation and MA by the Marie Sklodowska-Curie Actions (799929). M.J. is supported by The Australian Research Council's Discovery Early Career Researcher Award (DE190100565). F.A.M. is supported by an Australian National Health and Medical Research Council Senior Research Fellowship (GNT1060075). T.T. and A.T. are supported by the European Regional Development Fund (Project No. 2014-2020.4.01.15-0012), by Welcome Trust International Fellowship WT095077MA, by European Research Council grant GLIOGUIDE and Estonian Research Council (grants PRG230 and EAG79, to T.T.). Author contributions: G.B., M.S., A.H. conceived the project. L.C.C., R.O., L.D.P, M.D., J.F., S.K., F.v.d.M, K.K., M.A., L.S. designed and carried out experiments, A.T., T.T., L.L., O.V., J.H., O.G., H.K.K., P.O., M.J., developed and provided tools, L.C.C., R.O., L.D.P., M.D., J.F., S.K., T.K., C.S., T.S., M.J., F.A.M., S.J.B., J.H., O.V. analyzed the data or supervised data acquisition. L.C.C., R.O., L.D.P., M.D., J.F., S.K., T.K., O.G. visualized the data, T.K. and O.G. performed the scRNA-seq data analysis, M.S.W., B.M., C.S., H.K.K. provided human samples, G.B. and M.S. wrote the manuscript, G.B. and M.S. supervised the project. Competing interests: T.T., O.V. and G.B. have a patent on the monoclonal Antibody 3 (mAb3) against the NRP1 b1 domain for SARS-CoV-2 inhibition pending. Data and materials availability: All data needed to evaluate the conclusions in the paper are present in the paper or the Supplementary Materials. This work is licensed under a Creative Commons Attribution 4.0 International (CC BY 4.0) license, which permits unrestricted use, distribution, and reproduction in any medium, provided the original work is properly cited. To view a copy of this license, visit https://creativecommons.org/licenses/by/4.0/. This license does not apply to figures/photos/artwork or other content included in the article that is credited to a third party; obtain authorization from the rights holder before using such material.

\section{SUPPLEMENTARY MATERIALS}

science.sciencemag.org/cgi/content/full/science.abd2985/DC1

Materials and Methods

Figs. S1 to S11

Table S1

References (26-36)

MDAR Reproducibility Checklist

12 June 2020; accepted 12 October 2020

Published online 20 October 2020

10.1126/science.abd2985 

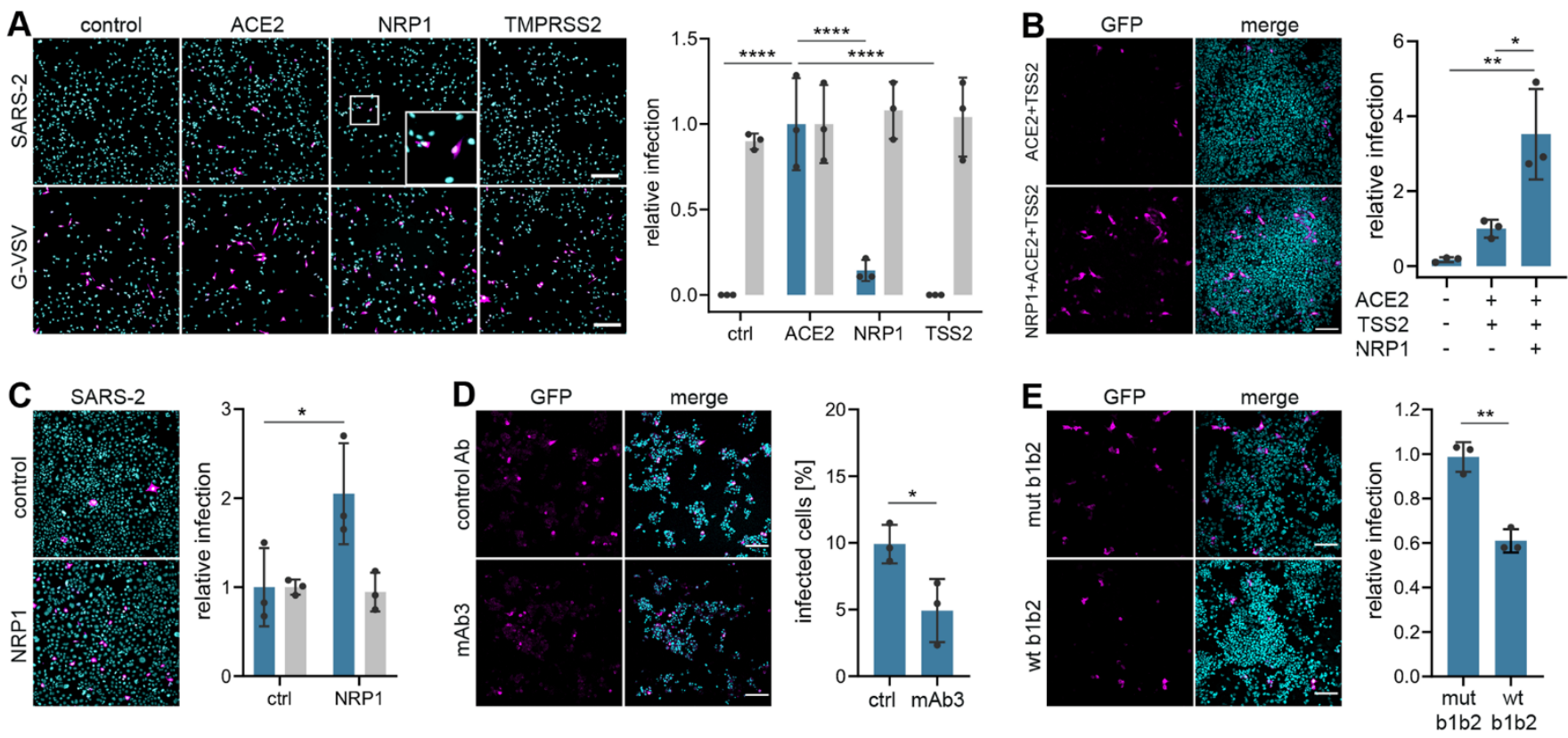

Fig. 1. NRP1 facilitates the cellular entry of SARS-CoV-2 pseudotyped particles. (A) Representative images and quantification of SARS-CoV-2 spike protein (SARS-2) (blue bars) and VSV-G pseudotype (gray bars) infectivity in HEK-293T cells transiently expressing control (ctrl) vector, ACE2, NRP1 or TMPRSS2 (TSS2). Data are normalized to the respective infectivity of SARS-2 and VSV-G pseudotype in ACE2-expressing cells. Twoway ANOVA with Tukey's correction for multiple comparisons. (B) HEK-293T cells transiently expressing ACE2 and TMPRSS2 or NRP1, ACE2 and TMPRSS2 were inoculated with SARS-2 pseudotype. Data are normalized to SARS-2 infectivity in cells expressing ACE2 and TMPRSS2. One-way ANOVA with Tukey's correction for multiple comparisons. (C) SARS-2 pseudotype infectivity in Caco-2 cells expressing NRP1 or control vector. Data are normalized to the respective infectivity of SARS-2 and VSV-G pseudotype in control cells. Two-way ANOVA with Sidak's correction for multiple comparisons. (D and E) HEK-293T cells transiently expressing NRP1, ACE2 and TMPRSS2 were inoculated with SARS-2 pseudotype in the presence of mAb3 antibody against NRP1 (D, mAb3) or control mAb2 (D, ctrl Ab), and in the presence of soluble NRP1 wild-type b1b2 domain (E, wt b1b2) or the NRP1 mutant b1b2 domain (E, mut b1b2). Data in (E) are normalized to untreated cells expressing NRP1, ACE2 and TMPRSS2. Two-tailed unpaired Student's $t$ test. All data are represented as mean \pm s.d. from three independent experiments ( $A$ to $C$ ) or three biological replicates ( $D$ and $E$ ). ${ }^{*} p<0.05,{ }^{*} p<$ $0.01,{ }^{* * * *} \mathrm{p}<0.0001$. All images show GFP-positive, infected cells (magenta) and Hoechst (cyan). Scale bars, $100 \mu \mathrm{m}$. 
A

\begin{tabular}{|c|c|c|}
\hline Sequence & Furin cleavage site & $\%$ \\
\hline NC_045512.2_Wuhan-Hu-1WT & ASYQTQTNSPRRAR^SVA & 100 \\
\hline FIN-UH25M-Nasopharynx & ASYQTQTNSPRRAR^SVA & 100 \\
\hline Finland/1/2020-Nasopharynx & ASYQTQTNSPRRAR^SVA & ND \\
\hline FIN-UH25M-Caco-2 P3§ & ASYQTQTNSPRRAR^SVA & 100 \\
\hline Finland/1/2020-VeroE6 P1 & ASY .... S SPRRAR^SVA & 53 \\
\hline Finland/1/2020-VeroE6 P3 & ASY - . . - SPRRAR^SVA & 78 \\
\hline Finland/1/2020-VeroE6 P7 §§ & ASY . . . . SPRRAR^SVA & 100 \\
\hline
\end{tabular}
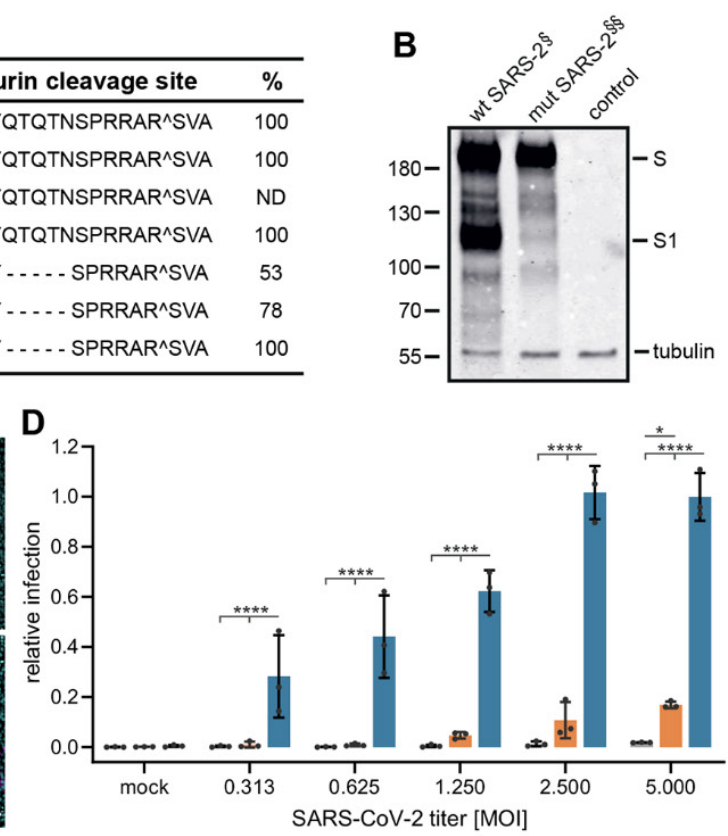

E $\quad$ ACE2

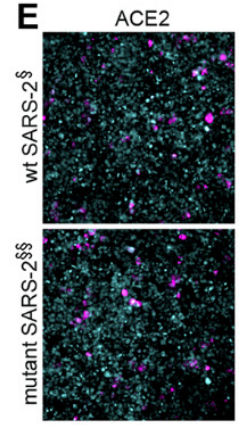

TSS2+ACE2

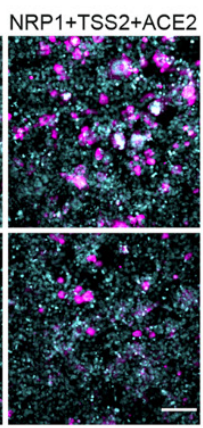

$F$
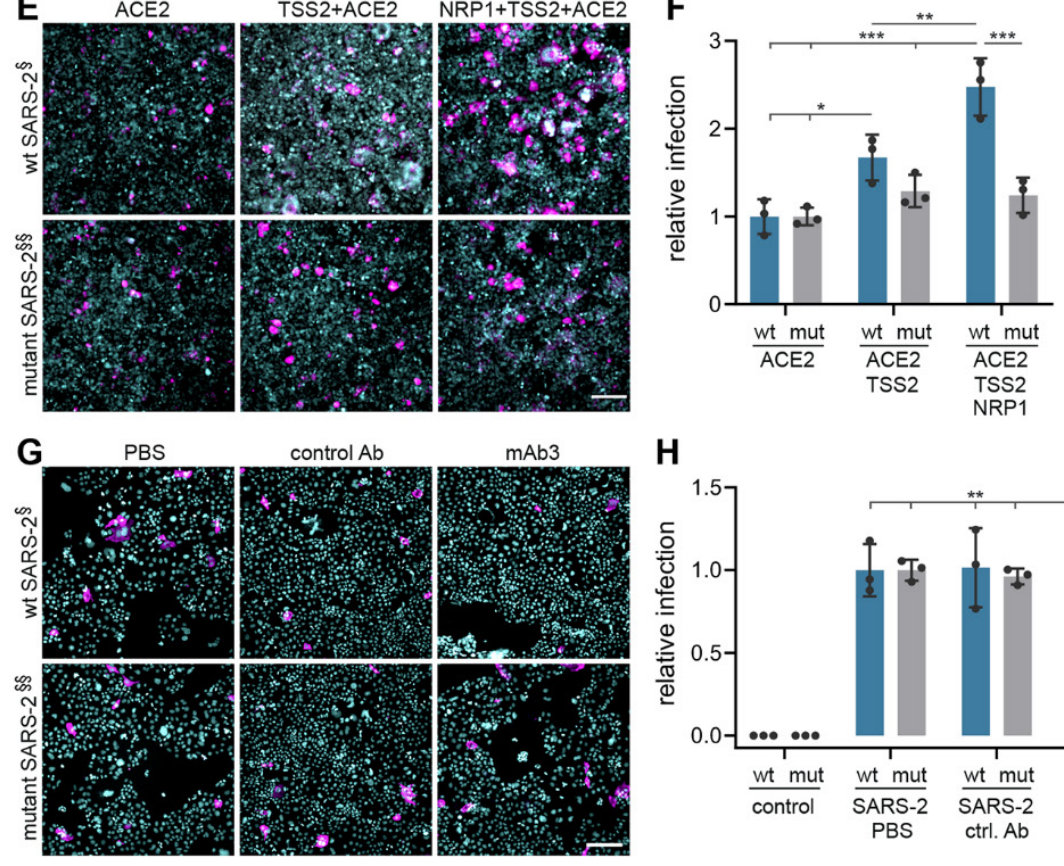

H

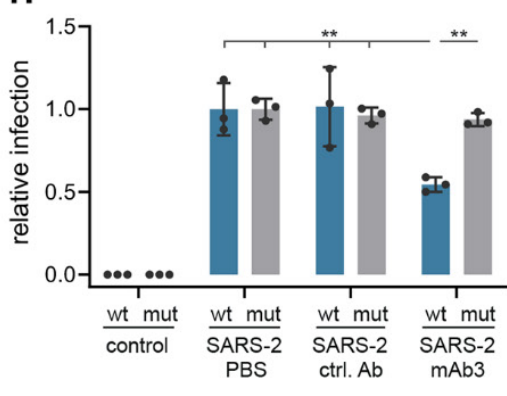

Fig. 2. A blocking antibody against the b1b2 domain of NRP1 reduces infection by wild-type SARS-CoV-2 (SARS2-wt), but not a mutant with a deletion at the furin-cleavage site (SARS-2-mut). (A) Sequence analysis of viruses isolated at different passages $(P)$ from different cell types. The first sequence is the reference from the Wuhan isolate (NC_045512.2). The sequence abundance in each virus population is indicated as a percentage (\%). $\S=$ SARS-2-wt, $\S \S=$ SARS-2-mut, used in the experiments. (B) A deletion adjacent to the furin-cleavage site abrogates the enzymatic cleavage of the $S$ protein. Immunoblot analysis of cell lysates from Vero-E6 cells infected for 16h with two viral populations ( $\S$ and $\S \S$ ). ( $C$ and $D)$ Representative images and quantification of SARS-2-wt infectivity in HEK293T cells stably expressing ACE2 (blue bars) or NRP1 (orange bars) compared to non-transfected cells (gray bars). Different virus titers were used. Data are normalized to the infectivity in ACE2-expressing cells at MOI 5. Two-way ANOVA with Tukey's correction for multiple comparisons. ( $E$ and $F$ ) Representative images (E) and quantification (F) of HEK-293T cells stably expressing the indicated combinations of ACE2, TMPRSS2 (TSS2) and NRP1 following SARS-2-wt (wt, blue bars) or SARS-2-mut (mut, gray bars) inoculation. Data are normalized to the respective infectivity in ACE2-expressing cells. Two-way ANOVA with Tukey's correction for multiple comparisons. (G and $H)$ Caco-2 cell infection in the presence of control mAb2 (ctrl. Ab) or mAb3 blocking antibodies against NRP1 after SARS-2-wt (wt, blue bars) or SARS-2-mut (mut, gray bars) inoculation. Data are normalized to the respective vehicle control (PBS) sample. Two-way ANOVA with Tukey's correction for multiple comparisons. Data are mean \pm s.d. from three independent experiments, ${ }^{*} p<0.05,{ }^{* *} p<0.01,{ }^{* *} p<0.001,{ }^{* * *} p<0.0001$. SARS-2-wt and SARS-2-mut infected cells (magenta), Hoechst (cyan). Scale bars, $50 \mu \mathrm{m}$. 
A

SARS-2 S protein

SARS-2-Ala SARS-2-pre SARS-2-post SARS-2-post amide

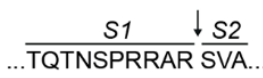

biot-X-TQTNSPRRAA-OH biot-X-TQTNSPRRAR^SVA-OH biot-X-TQTNSPRRAR-OH biot-X-TQTNSPRRAR-NH2
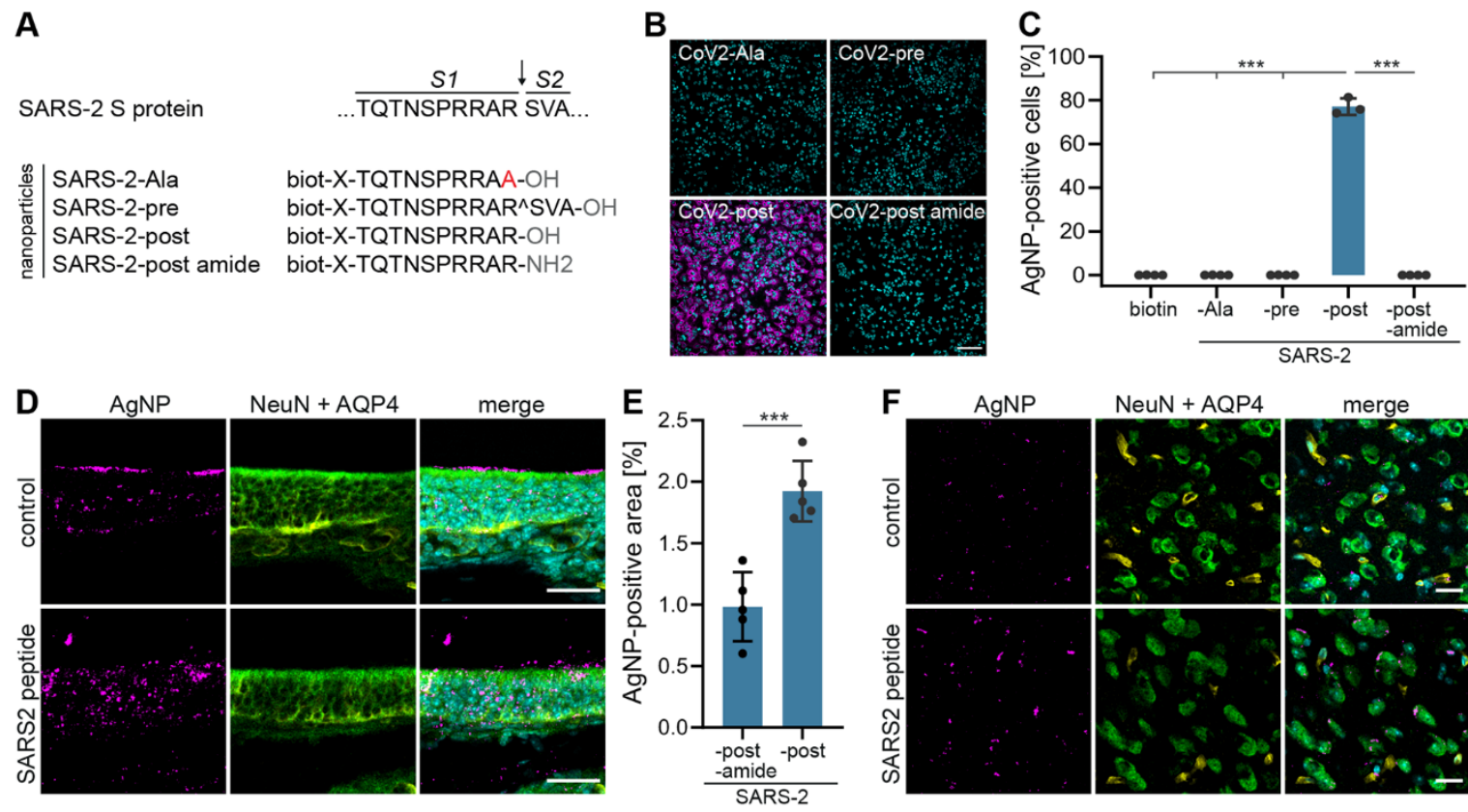

F $\quad \mathrm{AgNP} \quad \mathrm{NeuN}+\mathrm{AQP} 4$

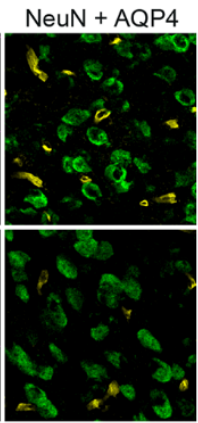

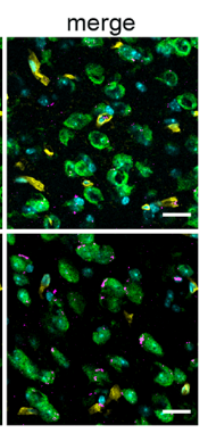

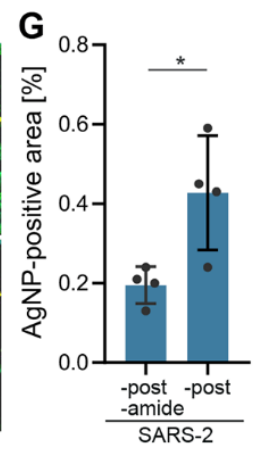

Fig. 3. NRP mediates entry of nanoparticles coated with SARS-2 $S$ derived CendR peptides into cultured cells, olfactory epithelium and CNS of mice. (A) Sequence of the peptides used for AgNP coating. Peptides that mimic SARS-2 S protein after furin cleavage (post) and as controls, S protein before cleavage (pre), with a point mutation of the terminal arginine (Ala) or with an amide terminus (post amide). (B and C) Representative images and quantification of the internalization of peptide-coated AgNPs in HEK-293T cells expressing NRP1. Merged images show AgNP-positive cells (magenta) and Hoechst (cyan). One-way ANOVA with Tukey's correction for multiple comparisons. ( $D$ to $G$ ) Representative images and quantification of main olfactory epithelium (MOE) ( $D$ and $E$, respectively) and cortex ( $F$ and $G$, respectively) 6 hours after intranasal administration of AgNPs coated with SARS2-post and SARS2-post amide peptides. $n=4$ replicates for $C ; n=5$ $(E)$ and $n=4(G)$ mice per condition. Data are means \pm s.d. Two-tailed unpaired Student's $t$ test ${ }^{*} p<0.05$, ${ }^{* * *} p$ $<0.001$. AgNPs (magenta), Hoechst (cyan), NeuN (neuronal marker, green), AQP4 (yellow). Scale bars, 100 $\mu \mathrm{m}(\mathrm{B}), 20 \mu \mathrm{m}(\mathrm{D}, \mathrm{F})$. 

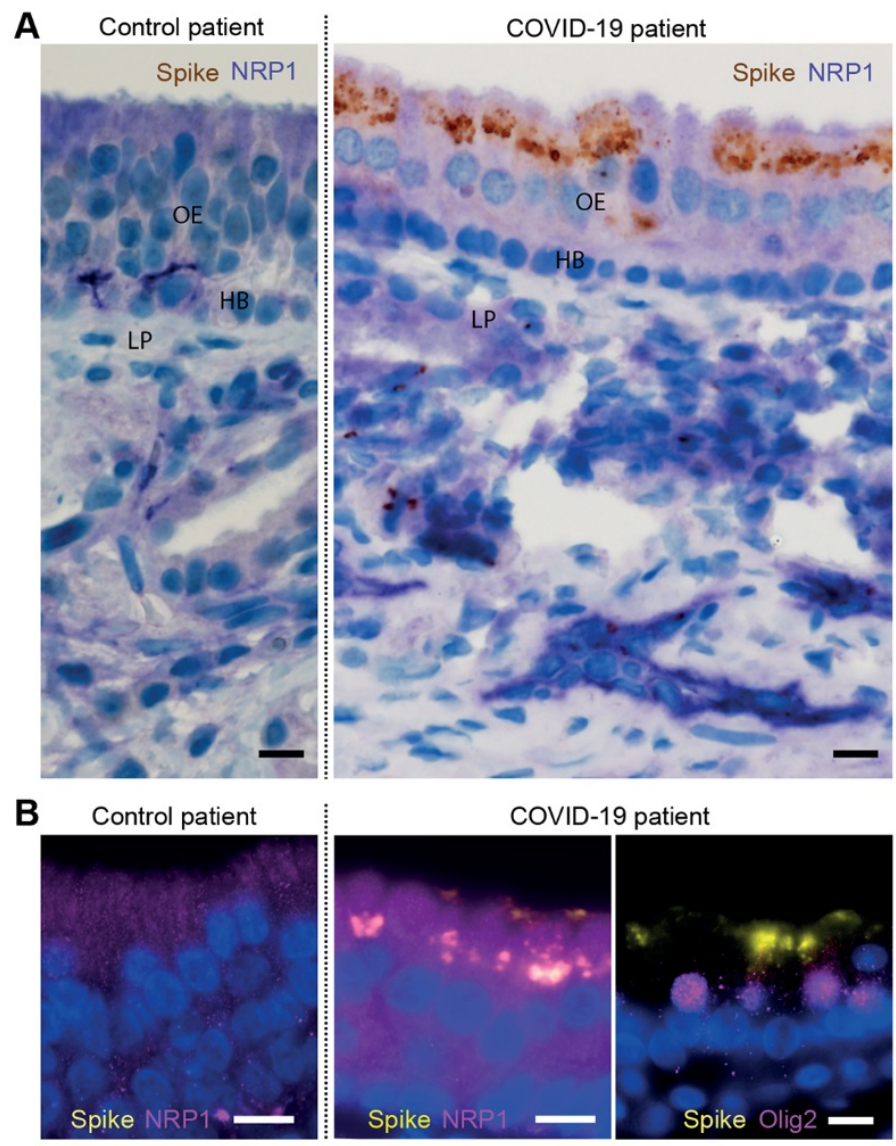

Fig. 4. SARS-CoV-2 infects the olfactory epithelium. (A) Co-staining of S protein (brown) and NRP1 (lilac) in the apical olfactory epithelium (OE) in a COVID-19 patient and non-infected control (LP, lamina propria, $\mathrm{HB}$, horizontal basal cells). (B) Co-localization of NRP1 (magenta) and $S$ protein (yellow) in OE cells in a COVID-19 patient. Co-staining of OLIG-2 (magenta) and $S$ protein (yellow) reveals infection of late olfactory neuronal progenitors/newly differentiated olfactory neurons. Scale bars, $10 \mu \mathrm{m}$. 